\title{
A New Approach to Identify the Fracture Zone and Detection of Bone Diseases of X-Ray Image
}

\author{
Tapan Kumar Hazra ${ }^{1}$, Subhadipa Dutta ${ }^{2}$ \\ ${ }^{1}$ Institute of Engineering \& Management, Department of Information Technology, \\ Y-12, Salt Lake Electronics Complex, Sector - V, Kolkata - 700 091, West Bengal, India \\ ${ }^{2}$ Institute of Engineering \& Management, Department of Information Technology, \\ Y-12, Salt Lake Electronics Complex, Sector - V, Kolkata - 700 091, West Bengal, India
}

\begin{abstract}
The present paper focuses on analysis of bone x-ray images to identify any abnormality zone for possible bone diseases or fractures. The proposed research work is useful in the field of bio-medical imaging. The proposed work involves image pre-processing steps such as denoising, histogram smoothing, segmentation and edge detection, in order to enhance the given image quality and convert it into denoised image to identify and separate the region of interest (ROI) manually using algorithms for calculating bone mineral density (BMD) value to analyse and identify bone diseases and fracture risk. In this work we have to calculate some Gray Level Co-occurrence Matrix (GLCM) features and other some more features like mean, median and standard deviation are also derived. At the final stage we have created a database of both the normal and eroded bone images by applying BMD and GLCM features for classification and comparison of values in order to determine whether the input image is infected or not.
\end{abstract}

Keywords: ANN, SVM, confusion matrix, accuracy, precision, recall and specificity

\section{Introduction}

The proposed work comes under bone imaging. Bone is a living, growing tissue which is mostly made of two materials: collagen (protein) and calcium (mineral), this combination makes human bone strong and flexible enough to hold up under stress. Initially as a person grow his bones also grow faster but after a certain age this process reverse, means start to lose a certain amount of bone material. Bones become less dense means the chances of bone diseases and fracture increases. If anyone has high bone mineral loss, then the most common disease that majority faces are diseases like osteoporosis, arthritis etc. Objective of our proposed work is to identify the fracture zone if any fracture exist, else trying to identify whether the bone is affected by osteoporosis or not. In the present paper, we have proposed a new approach to identify the Fracture zone and detection of Bone Diseases within a same working model of an x-ray Image by applying some mathematical formula. In this context it is wise to note Osteoporosis is degenerative disease and arthritis is an autoimmune disease that mainly affects the joints in the human body.

Here first we have to convert the raw image into a noise free image using some pre-processing technique. Now we have to select our focused area of interest which is named as region of interest (ROI). After getting this ROI we can derive some Gray Level Co-occurrence Matrix (GLCM) features like contrast, correlation, homogeneity, energy and some common features like mean, standard deviation, area etc. for classification. In the next step using those extracted values we can classify whether bone is affected by diseases or not through artificial neural network (ANN). Although ANN is a good classifier and gives good accuracy but Support Vector Machines (SVM) is often superior to ANNs because they avoid two major weaknesses of ANN. Two common and major weaknesses are:
1)ANNs often converge on local minima rather than global minima, meaning that they are essentially "missing the big picture".

2)ANNs often over fit if training goes on too long, meaning that for any given pattern, an ANN might start to consider the noise as part of the pattern.

We can also conclude by comparing both classifier output which one is superior to other by classifying its accuracy or error rate. Here performance of proposed work is evaluated in terms of accuracy, precision, recall and specificity. For calculating those values, first we have to form one confusion matrix also known as contingency table. This confusion matrix contains true positive, true negative, false positive, false negative values. Here we compare values of four parameter for same image using two different classifiers. First we have to select Region of interest then form this area using mathematical formula; we have to calculate different types of feature values. In the next step we have to create two data set for all collected image samples; one for inputs and another for targets. Now using neural network feed forward back propagation algorithm we have to import and train with these data. In the next step it will automatically generate one page which contain some option for output measurement like performance graph, confusion matrix etc. and from this confusion matrix we can calculate the accuracy, recall, specificity, precision value using mathematical formula. In next step, using another classifier support vector machine (SVM) we can calculate those accuracy, recall, specificity, precision using same formula of the same image. Now we can compare both values to conclude which classifier is more suitable.

\section{Literacy Survey for Related Work}

In general we use DEXA i.e. Dual-energy X-ray absorptiometry for measurement of Bone mineral density. 


\section{International Journal of Science and Research (IJSR) \\ ISSN (Online): 2319-7064}

Index Copernicus Value (2013): 6.14 | Impact Factor (2015): 6.391

Jiang et al. (2010) worked on medical image processing using artificial neural network [4]. The techniques and algorithms were explained in detail to provide inspiring examples illustrating: (i) how a known neural network with fixed structure and training procedure could be applied to resolve a medical imaging problem; (ii) how medical images could be analyzed, processed, and characterized by neural networks; and (iii) how neural networks could be expanded further to resolve problems relevant to medical imaging. In the concluding section, a highlight of comparisons among many neural network applications is included to provide a global view on computational intelligence with neural networks in medical imaging.

Bone mineral density can also be measured from the images those are obtained Quantitative Computed Tomography (QCT), Qualitative Ultrasound (QUS), Single Photon Absorptiometry (SPA), Dual Photon Absorptiometry (DPA), Digital X-ray Radiogrammetry (DXR), Single energy X-ray Absorptiometry (SEXA). Hypophosphatasia, Klippel-Feil syndrome, Craniosynostosis, Fibrous dysplasia, and Osteoarthritis are the diseases that affect bones of a human body and decrease the bone mineral density. Many research works are done related to bone mineral density and Gray Level Co-occurrence Matrix .In our proposed work we are mainly focused to detect osteoporosis and arthritis more accurately osteoarthritis and also trying to identify the fracture zone .

Lim, Sher Ee, et al. (2004) worked on same topic but specifically femur and radius fractures [5]. They describe an approach in detecting fractures of the femur and the radius by combining various detection methods. These methods extract different kinds of features for fracture detection.

They include neck-shaft angle, which is specifically extracted for femur fracture detection, and Gabor texture, Markov Random Field texture, and intensity gradient, which are general features that can be applied to detect fractures of various bones. Two types of classifiers are tested, namely, Bayesian classifier and Support Vector Machine. Test results show that the combined approach can improve both the fracture detection rate and the classification accuracy significantly compared to any single method.

Lum et al. (2005) was also worked on x-ray image to identify the fracture zone. In medical applications, sensitivity in detecting medical problems and accuracy of detection are often in conflict. A single classifier usually cannot achieve both high sensitivity and accuracy at the same time. Methods of combining classifiers have been proposed in the literature. This paper presents a study of probabilistic combination methods applied to the detection of bone fractures in x-ray images. Test results show that the effectiveness of a method in improving both accuracy and sensitivity depends on the nature of the method as well as the proportion of positive samples.

Umadevi and Geethalakshmi (2012) studied on bone structure. They improved the segmentation method for diaphysis extraction from x-ray images. A model that combines multi-resolution wavelets, region growing algorithm and active contour model is proposed to segment the bone structure from the $x$-ray image [6]. They use a fast Hough transformation to extract the diaphysis region from the segmented bone structure. They concluded that the proposed algorithm is efficient both in the manner of segmentation and speed of segmentation.

Chokkalingam and Komathy (2014) worked on rheumatoid arthritis and bone mineral density using Gray Level Co-occurrence Matrix (GLCM) [7]. Their dataset is trained with inflamed and non inflamed values and with the help of neural network all the new images are checked properly for their status and rough set is implemented for further reduction.

Vinoth and Jayalakshmi (2014) estimate the bone mineral density using digital X-Ray images for detection of rheumatoid arthritis [3]. They worked on bone image and the objective of their study is to detect the occurrence of Rheumatoid Arthritis (RA). Their work involves steps such as denoising, histogram smoothing, segmentation and edge detection in order to enhance the given image and separate the region of interest. The strength of a bone depends on Bone Mineral Density (BMD) which is a major factor in identifying bone diseases and fracture risk. The mathematical relationship between Bone Mineral Content and Volume by Region of Interest will help in calculating Bone Mineral Density and various features of bone images such as area, mean, standard deviation and variance. The Gray Level Co-occurrence Matrix (GLCM) is one of the image analysis techniques that can be used for extracting features (Energy, Entropy, Contrast, Homogeneity and Correlation) of a bone image. They created a dataset from both the normal and eroded bone images by applying BMD and GLCM features. Whenever a new bone image is given as an input, the features of the image are extracted and compared against the dataset. The input image is classified as whether infected or not infected using neural network which achieved classification accuracy of about $96.66 \%$.

\section{System Design and Implementation}

$\mathrm{X}$-ray bone images are collected from various sources and we have to create one database with the values of extracted features of the selected images. The main objective of the proposed system is to analyse the Bone Mineral Density (BMD) present at the Region of Interest (ROI) and according to this BMD and other features value we have to classify whether the image is effected by any bone diseases like osteoporosis or not and one more approach is also added here that is identify the fracture zone or risk of fracture. For implementing the whole concept we suggest one working model the below flow chart describe the procedure how to proceed step by step 


\section{International Journal of Science and Research (IJSR) \\ ISSN (Online): 2319-7064}

Index Copernicus Value (2013): 6.14 | Impact Factor (2015): 6.391

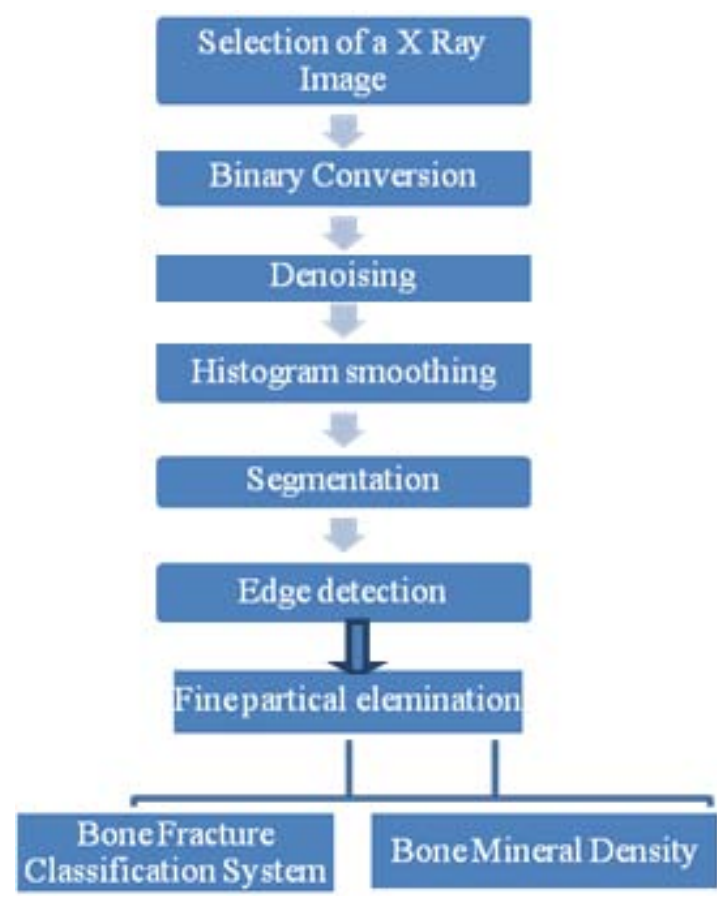

Figure 1: The flow chart describes the $x$-ray image processing for fracture detection and the bone mineral density estimation

Step-1: Selection of An X-ray image: - The selection of the image is the most vital part and the image may be a fracture image or osteoporosis affected image or be it a normal image .One more thing we have to strictly follow is that, all images we collect must be of same type with same specification.

Step-2: Binary conversion: The image is changed to binary to ease the computing process and maximize the speed of calculating due to the rapid Boolean operators. By changing to binary, a bone (femur) shaft can be separated from the soft tissue shade which can be considered noise during the bone shaft image processing.

Step-3: Denoising-The main properties of a good image denoising model is that it will remove noise while preserving edges. Traditionally, linear models have been used. One common approach is to use a Gaussian filter, or equivalently solving the heat-equation with the noisy image as input-data, i.e. a linear, $2^{\text {nd }} \mathrm{PDE}$-model.

Step-4: Histogram smoothing:- An important application of histogram-based filters is in edge preserving smoothing. Often, such filters are used to decompose images into the sum of a base layer and a detail layer for further processing. For this purpose, an important limitation is that histogram-based filters often increase the sharpness of the edges they preserve.

Step-5: Segmentation:-. A popular method used for image segmentation is thresholding. After that a binary image is formed, where all object pixels have one gray level and all background pixels have another -generally the object pixels are 'black' and the background is 'white'. The best threshold is the one that selects all the object pixels and maps them to 'black'
Step-6: Edge detection: Edge detection is the process of localizing pixel intensity transitions. Edge detection algorithm can detect edges in image even if it contains noise which refers to noise sensitivity. Canny which is a popular edge detection method is considered in this work.

Step-7: Fine practical elimination: By using the median filter algorithm, the target pixel's value is replaced by the median value of the neighboring pixel in a kernel. The kernel is of importance in determining output image. These are the algorithm parameters that should carefully chosen depending on the image fine particles. The median filter is the filter chosen after the edge detector due to its ability to suppress isolated noise while preserving the femur bone border edge.

After completing the pre-processing step we move to the next process for selection of ROI.

In our proposed system other steps are created in an automatic manner. Initially region of interest is selected by drawing ellipse and then selected portion is segmented. Then it is mapped with white color and again selected portions are mapped with black color and stored as new image. Finally these white and black images are subtracted in order to identify region of interest and stored as a new image. From this portion of region of interest are identified clearly and accurately. Finally edges are detected using Prewitt algorithm from ROI.

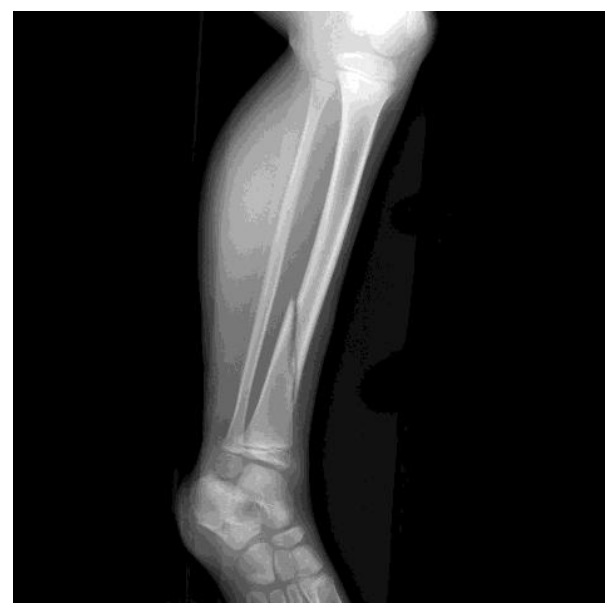

Figure 2: Original image

After completing the pre-processing and focused on selected ROI

Volume 5 Issue 9, September 2016

www.ijsr.net

Licensed Under Creative Commons Attribution CC BY 


\section{International Journal of Science and Research (IJSR) \\ ISSN (Online): 2319-7064}

Index Copernicus Value (2013): 6.14 | Impact Factor (2015): 6.391

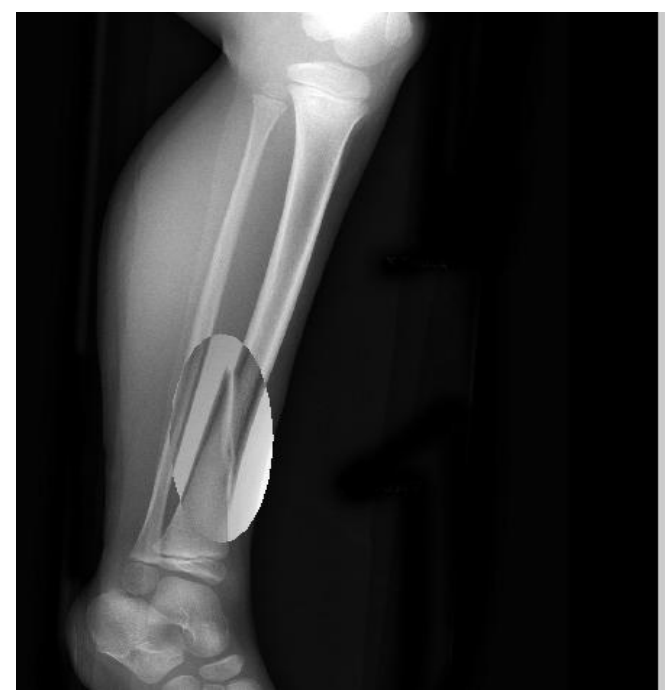

Figure 3: Inversion of the segmented portion

In this step we inverse the ROI portion for better analysis and identification of the fracture zone

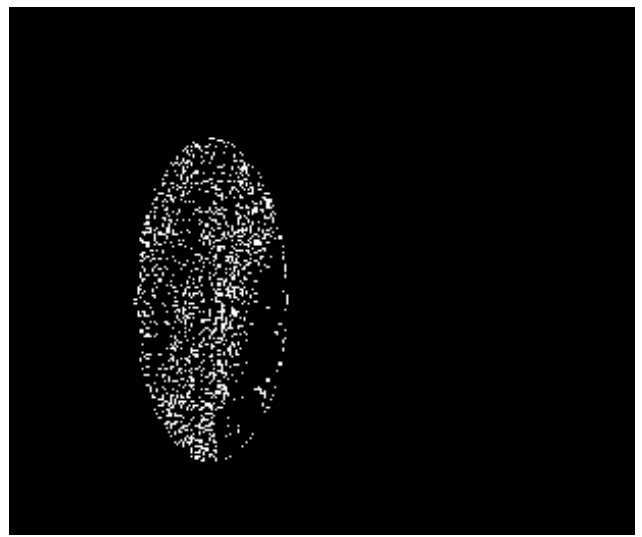

Figure 4: Edge detected image using Prewitt algorithm

\subsection{Bone Mineral Density Calculation and Feature Extraction}

BMD: Bone mineral density (BMD) test can provide a snapshot of bone health. BMD test measures how much calcium and other types of minerals are in an area of your bone. The test can identify osteoporosis, determine your risk for fractures (broken bones), and measure response to osteoporosis. The more dense the bones (from greater mineral content), the more energy is absorbed, and the less energy detected. The BMD test is an important predictor of whether a person will have a risk of developing any fracture in future. A BMD test measures your bone mineral density and compares it to that of an established norm or standard to give you a score. Although no bone density test is 100 -percent accurate.

\section{Bone mineral density (BMD) tests are used to:}

1) Diagnose bone loss and osteoporosis types bone diseases.

2) See how well osteoporosis medicine is working.

3) Predict your risk of future bone fractures.

Most commonly, your BMD test results are compared to the ideal or peak bone mineral density of a healthy 30-year-old adult and you are given a T-score. A score of 0 means your $\mathrm{BMD}$ is equal to the norm for a healthy young adult. Differences between your BMD and that of the healthy young adult norm are measured in units called standard deviations (SDs). The standard deviations below 0 , indicated as negative numbers, the lower your BMD and the higher your risk of fracture and osteoporosis.

Table 1: Universal value of Standard Deviation for BMD calculation

\begin{tabular}{|c|c|}
\hline LEVEL & DEFINITION \\
\hline NORMAL & $\begin{array}{c}\text { Bone density is within } 1 \text { SD }(+1 \text { or }-1) \text { of the } \\
\text { young adult mean }\end{array}$ \\
\hline $\begin{array}{c}\text { LOW BONE } \\
\text { MASS }\end{array}$ & $\begin{array}{c}\text { Bone density is between } 1 \text { and } 2.5 \text { SD below the } \\
\text { young adult mean }(-1 \text { to }-2.5 \text { SD). }\end{array}$ \\
\hline OSTEOPORESIS & $\begin{array}{c}\text { Bone density is } 2.5 \text { SD or more below the young } \\
\text { adult mean }(-2.5 \text { SD or lower). }\end{array}$ \\
\hline $\begin{array}{c}\text { SEVERE } \\
\text { (ESTABLISHED) } \\
\text { OSTEOPORESIS }\end{array}$ & $\begin{array}{c}\text { Bone density is more than } 2.5 \text { SD below the } \\
\text { young adult mean, and there have been one or } \\
\text { more osteoporotic fractures. }\end{array}$ \\
\hline
\end{tabular}

We have followed a new mathematical relationship and procedure to calculate bone mineral density from the region of interest. Calculation of Bone Mineral Density can be performed using following mathematical relationship.

\section{Bone Mineral Density $=$ Bone Mineral Content/volume of roi}

Bone mineral content $=($ Sum of volume of pixels in roi*1.073)/Calibration of ROI

Volume of ROI $=\pi\left(\frac{\text { Diameter }}{2}\right) \wedge 2 * K$

Bone Mineral Density is derived from Bone Mineral Content (BMC) of the region of interest and volume of region of interest. BMD is measured in grams/cm3, BMC is measured in grams and volume of ROI is measured in $\mathrm{cm}^{3}$. Sum of volume of pixels in ROI refers to the integrated density of ROI. 1.073 grams is the default value for all the images that refers to the amount of air and water present in bone. Volume of ROI is measured using above mentioned formula. In that formula, diameter is calculated from area of region of interest and $\mathrm{k}$ refers entropy of the image. Using some existing tools and algorithms, we can calculate the values of integrated density, area, perimeter etc.

In this present study the Gray Level Co-occurrence Matrix (GLCM) is used for the estimation of BMD. GLCM is one of the very famous and popular image analysis techniques which are used to extract above mentioned texture features and classification.

GLCM is main tool used in image texture analysis. GLCM is a statistical way to indicate image texture structure by statistically sampling the pattern of the grey levels occurs in relation to other grey levels. In GLCM Correlation refers that positively or negatively a pixel is correlated with neighbor pixel. Contrast refers to intensity contrast or brightness of a pixel with its neighbor pixel over whole image. In other way, in a continuous set of pixels, difference between highest and lowest values is contrast. Uniformity of pixels pairs or similarity between pixels is called as energy. Homogeneity returns the values of GLCM to GLCM diagonal by applying closeness distribution of elements.

\section{Volume 5 Issue 9, September 2016




\section{International Journal of Science and Research (IJSR) \\ ISSN (Online): 2319-7064}

Index Copernicus Value (2013): 6.14 | Impact Factor (2015): 6.391

In this work, some more features like Mean, Median, Variance and Standard deviation are also derived for better and more accurate classification for both normal and eroded bone images. These values are used for classification because these values will be different for normal and eroded bone images. New procedure is created for calculating Mean, Median, Variance and Standard deviation. Calculation of these features can be performed in this manner. For calculating mean value, intensity value of each pixel are added and divided by number of pixel in region of interest. For calculating median value, each pixel values are arranged in ascending order. If the count of pixel is odd number, middle value from that ascending order consider as median value. If the count of pixel is even number, then middle two values are taken and average of the two values is considered as median value.

Variance and standard deviation of region of interest can be calculated. Here we calculate mainly 11 important features .
1.Energy
2.Entropy
3. Correlation
4. .Homogeneity
5. Contrast
6. Mean
7. Standard deviation
8. Bone Mineral Density
9. Integrated Density
10. Area
11. Perimeter.

For classification, after completing the features calculation from ROI we move to the neural network for classification. Also create two sets of data one as input data and other data set as target data.

\subsection{Classification using Neural Network}

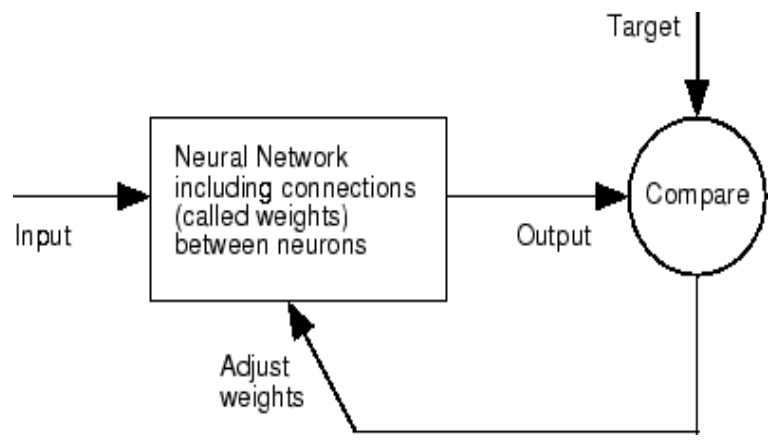

Figure 5: Neural network working principal

In the present work a dataset is created from both the normal and eroded bone images by applying BMD and GLCM features. Whenever a new bone image is given as an input, the features of the image are extracted and compared against the dataset. The input image is classified as whether infected or not infected using neural network. In this work, neural network is used for classification which gives good accuracy when compared against other classifier. Neural network is organized as layer and perception. There are three layers such as input layer, hidden layer and output layer. Input layer is passive mode and it takes input from outside and simply passed into hidden layer. Hidden layer is active mode and it has many functions such as weighted function which refers to the number of nodes inside the hidden layer and bias function which is used to fix the threshold value for network and Sigmoid function which is used to active the neural network. Finally output layer is in active mode and it is used to produce the output values as binary. In this proposed system we use feed forward back propagation algorithm as training or learning algorithm which learns by example. If you submit to the algorithm the example of what you want the network to do, it changes the network's weight so that it can produce desired output for a particular input on finishing the training. Here single layer perceptron is taken as classifier which is implemented with hard limit as transfer function and training rule with weighted/bias rule. Typically, neural networks are adjusted, or trained, so that a particular input leads to a specific target output.

After train using neural network we will get different types of analytical parameter like performance, Training state, error histogram, confusion matrix, Receiver operating characteristic. Here we consider two important analytical parameter those are performance graph and confusion matrix.

\section{Performance graph}

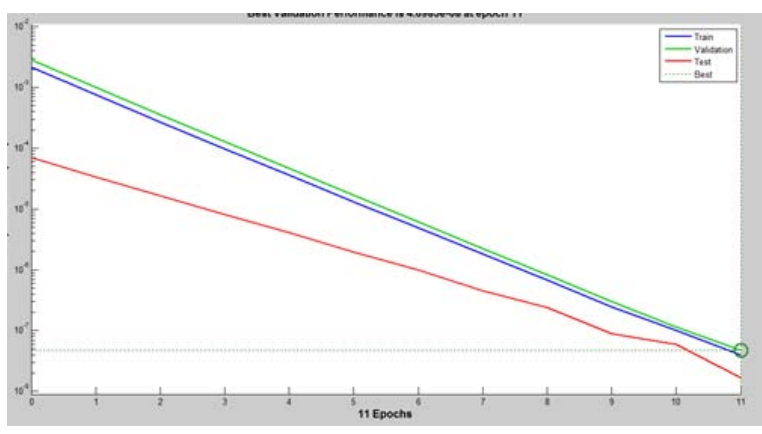

Figure 6: Performance graph

In this graph blue line indicates train data, green indicates validataion data graph, and red indicates test data.This figure does not indicate any major problem with the training if the validation and test curves are very similar.

From the output of confusion matrix we can calculate. prediction outcome

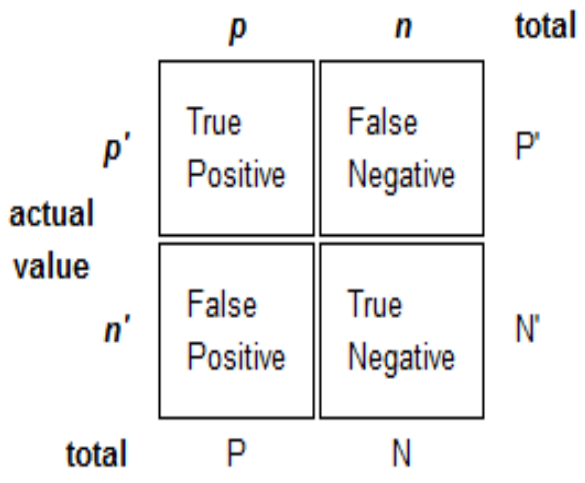

Figure 7: Confusion matrix 


\section{International Journal of Science and Research (IJSR) \\ ISSN (Online): 2319-7064}

Index Copernicus Value (2013): 6.14 | Impact Factor (2015): 6.391

True positives (TP): These are cases in which we predicted yes (they have the disease), and they do have the disease.

True negatives (TN): We predicted no, and they don't have the disease.

False positives (FP): We predicted yes, but they don't actually have the disease. (Also known as a "Type I error.")

False negatives (FN): We predicted no, but they actually do have the disease. (Also known as a "Type II error.").

Experimental output of our selected image:

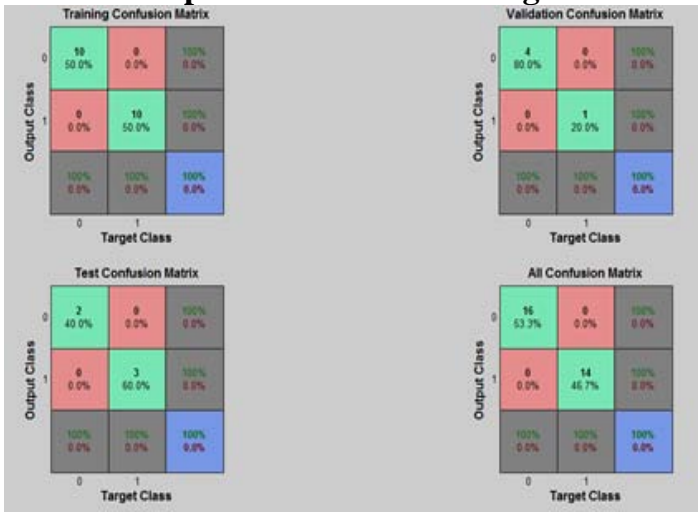

Figure 8: Confusion matrix

The network outputs are very accurate, as you can see by the high numbers of correct responses in the green squares and the low numbers of incorrect responses in the red squares. The lower right blue squares illustrate the overall accuracies.

The accuracy (AC) is the proportion of the total number of predictions that were correct. It is determined using the equation:

\section{ACCURACY $=(\mathbf{T P}+\mathbf{T N}) /(\mathbf{T P}+\mathbf{T N}+\mathbf{F P}+\mathbf{F N})$}

Output $=100 \%$ according to experimental values.

SENSITIVITY $($ RECALL $)=($ TP $) /($ TP+FN $)$

Output $=40 \%$ according to experimental values

PRECISION $=(\mathbf{T P}) /(\mathrm{TP}+\mathrm{FP})$

Output $=100 \%$ According to experimental values

\section{SPECIFICITY $=($ TN $) /(T N+F P)$ \\ Output $=100 \%$}

Although neural network is a good classifier then also in case of huge numbers some draw backs present here related to false positive and false negative in another word type-I and type-II error. Another classifier is support vector machine (Svm) which help to minimize this two type error gives better accuracy for this proposed work and chances of misclassification is tends to zero.

\subsection{Support Vector Machine (SVM)}

A Support Vector Machine (SVM).the operation of the SVM algorithm is based on finding the hyper plane that gives the largest minimum distance to the training examples

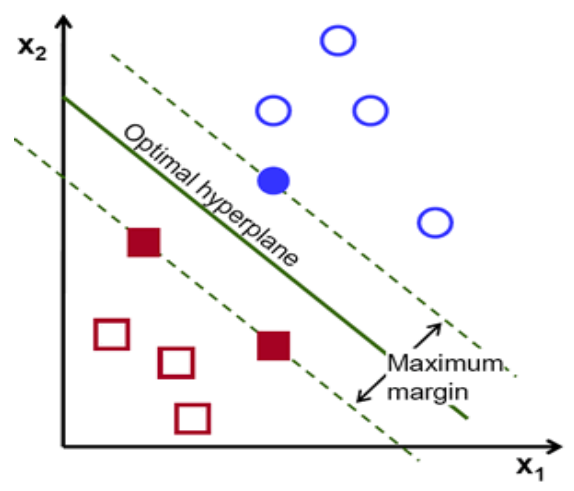

Figure 9: Support vector machine

In general, the training examples that are closest to the hyperplane are called support vectors. This representation is known as the canonical hyperplane.

\section{Classification Procedure:}

Step1: Set up the training data

Step2: Set up SVM's parameters

Step3: Train the SVM

Step4: Regions classified by the SVM

Step5: Identification of support vectors

Here we have used $\mathrm{R}$ visual studio for windows and $\mathrm{R}$ language for training and testing data. After the analysis the output we got is a confusion matrix. Using these results of confusion matrix we can calculate accuracy, sensitivity, Precision, Specificity.

From table2, we conclude that support vector machine is a better classifier then artificial neural network for all the four value. Here we consider less number of demo data, so analysis between two classifier is less. But in case of huge data we can observe that SVM gives more accurate result.

Table 2

\begin{tabular}{|c|c|}
\hline ANN & SVM \\
\hline $\begin{array}{c}\text { ACCURACY } \\
\text { Output }=\mathbf{1 0 0 \%}\end{array}$ & $\begin{array}{c}\text { ACCURACY } \\
\text { Output }=\mathbf{1 0 0 \%}\end{array}$ \\
\hline $\begin{array}{c}\text { SENSITIVITY(RECALL) } \\
\text { Output }=\mathbf{4 0 \%}\end{array}$ & $\begin{array}{c}\text { SENSITIVITY(RECALL) } \\
\text { Output }=\mathbf{1 0 0 \%}\end{array}$ \\
\hline $\begin{array}{c}\text { PRECISION } \\
\text { Output }=\mathbf{1 0 0 \%}\end{array}$ & $\begin{array}{c}\text { PRECISION } \\
\text { Output }=\mathbf{1 0 0} \%\end{array}$ \\
\hline SPECIFICITY Output=100\% & SPECIFICITY Output $=\mathbf{1 0 0} \%$ \\
\hline
\end{tabular}

Example:

\begin{tabular}{|l|l|l|l|l|l|l|l|}
\hline Number of & $\begin{array}{l}\text { Type of } \\
\text { neural } \\
\text { network }\end{array}$ & TP & FP & TN & FN & $\begin{array}{l}\text { SN(Sensitivity) } \\
\text { in \% }\end{array}$ & $\begin{array}{l}\text { SP(specificity) } \\
\text { in \% }\end{array}$ \\
\hline $\begin{array}{l}42(35 \\
\text { osteoporotic+7 } \\
\text { nomal) }\end{array}$ & $\begin{array}{l}\text { Feed } \\
\text { forward }\end{array}$ & 34 & 1 & 6 & 1 & 95.2 & 85.714 \\
\hline
\end{tabular}

\section{Conclusion and Future Work}

We proposed a method for automated femur bone fracture detection using GLCM computerized techniques. From this 


\section{International Journal of Science and Research (IJSR) \\ ISSN (Online): 2319-7064}

Index Copernicus Value (2013): 6.14 | Impact Factor (2015): 6.391

method we are able to classify the absence and presence of bone fracture based on the obtained parameter value from GLCM value and other features. The threshold bordering, the absence and presence of bone fracture will be set to a value. The accuracy of the developed algorithm will be achieved which will help to recognize the bone fracture automatically. A new scheme will be implement to diagnose the presence of rheumatoid arthritis by a series of image processing techniques which have been termed to be more effective than the other methods which perform the same task and hence provide a more effective approach in computer aided diagnosis. This is a huge step in the medical field and can be utilized further. The system may be further enhanced by the improvement of the edge detection as well as finding a better segmentation technique. Gray level co-occurrence matrix (GLCM) features like Mean, Median, Energy, Correlation, Bone Mineral Density (BMD) and etc. After finding all the features it stores in the database. Whenever a new bone image is given as an input, the features of the image are extracted and compared against the dataset. The input image will be classified in next term, whether image infected or not infected using neural network. In this work, neural network is used for classification which gives good accuracy when compared against other classifier. Although neural network gives a good accuracy in classification then also for more better classification we propose to classify using support vector machine where chance of misclassification near to zero. In future using fuzzy logic we will try to calculate percentage of infection.

\section{References}

[1] Chai, Hum Y., Lai K. Wee, Tan T. Swee, Sh-Hussain Salleh, A. K. Ariff, and R. Kamarulafizam. "Gray-level co-occurrence matrix bone fracture detection." American Journal of Applied Sciences 8, no. 1 (2011): 26.

[2] Harrison, B et al. "Assessing Periarticular Bone Mineral Density in Patients with Early Psoriatic Arthritis or Rheumatoid Arthritis." Annals of the Rheumatic Diseases 61.11 (2002): 1007-1011. PMC. Web. 18 Aug. 2016.

[3] Vinoth, M. andJayalakshmi, B. (2014). Bone Mineral Density Estimation Using Digital X-Ray Images for Detectionof Rheumatoid Arthritis. Int J Pharm Bio Sci 2014 July ; 5 (3) : (B) 104 - 121).

[4] Jiang, Jianmin, P. Trundle, and Jinchang Ren. "Medical image analysis with artificial neural networks." Computerized Medical Imaging and Graphics 34.8 (2010): 617-631.

[5] Lim, Sher Ee, et al. "Detection of femur and radius fractures in x-ray images." Proc. 2nd Int. Conf. on Advances in Medical Signal and Info. Proc. Vol. 65. 2004.

[6] Umadevi, N., and S. N. Geethalakshmi. "Enhanced Segmentation Method for bone structure and diaphysis extraction from x-ray images." International Journal of Computer Applications 37.3 (2012): 30-36.

[7] Chokkalingam, S. P., and K. Komathy. "Intelligent Assistive Methods for Diagnosis of Rheumatoid Arthritis Using Histogram Smoothing and Feature Extraction of Bone Images." World Academy of Science, Engineering and Technology International Journal of Computer, Control, Quantum and Information Engineering 8.5 (2014).

\section{Author Profile}

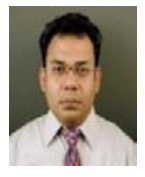

Tapan Kumar Hazra completed his M.E degree from Jadavpur University, Kolkata, West Bengal, India. Since from 2003, he is working as Assistant Professor of Department of Information Technology at Institute of Engineering \& Management, Salt Lake, Kolkata, West Bengal, India. His research interest include Design and Analysis of Algorithms, Image Processing, Natural Language Processing, Sentiment Analysis, Machine learning, Cryptography.

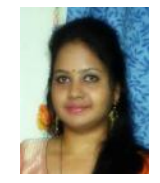

Subhadipa Dutta is pursuing M.Tech in Information Technology from Institute of Engineering and Management, Salt Lake, Kolkata, West Bengal, India. Her research interests include Medical Image Processing. 